\title{
ON LONGITUDINAL EMITTANCE MEASUREMENTS IN THE BOOSTER
}

\section{BOOSTER TECHNICAL NOTE \\ NO. 212}

\author{
D.-P. DENG
}

September 22, 1992 


\title{
On Longitudinal Emittance Measurements in the Booster
}

\author{
D.-P. Deng \\ Brookhaven National Laboratory \\ Upton, NY 11973
}

September 22, 1992

\begin{abstract}
Heavy ion beams $\left(S i^{8+}\right.$ and $\left.A u^{33+}\right)$ were accelerated and coalesced in the Booster with two Band III cavities $(2.4 \mathrm{M} \mathrm{Hz}-4.5 \mathrm{M} \mathrm{Hz})$ during runs in spring of 1992. Longititudianl emittance measurements are documented throughout the cycle in an effort to record the Booster performances for the future RHIC operations.
\end{abstract}

\section{Introduction to Longitudinal Emittance}

As the Booster is geared to deliver bunched proton and heavy ion beams for $R H I C$ operation in the near future, the importance of the longitudinal emittance has come of age. Unlike for fixed target experiments, colliding beam experiments require very short bunches. Small longitudinal emittance can provide short bunches.

The longitudinal direction is the direction the beam travels. The longitudinal emittance of a bunched beam basically describes the extent of the spread in the longitudinal bunch length and energy spread within the bunch.

The basic idea for the operation of the Booster is that bending magnetes hold the beam in the Booster ring and the cavities bunch and inject energies to the beam. To accelerate a bunch of charged particles, the fields of bending magnets are raised to higher fields, the particles then must pick up sufficient energies from the cavity gaps in order to keep up with the rising of bending magnetic fields. The bending magnets and the cavities can work in synchronism to maintain a reference or synchronous particle on a fixed reference orbit in the ring, others particles' orbits oscilate around the reference orbit. The synchronous particle gains the right amount of energy from the cavities to stay on the reference orbit, other particles gain more or less energies from the cavities depending on their positions. So the energy and the position of a particle are the variables to describe the motion in the longitudinal direction.

The longitudinal phase space is spanned by two canonical variables $\left(\frac{\Delta E}{\omega_{r f}}, \phi\right)$, where $\omega_{r f}$ is the angular frequency of the RF cavities and $\Delta E$ is the energy deviation from the synchronous particle energy $E$, determined by the state of the accelerator, and $\phi$ is the phase angle of 
the voltage across the RF cavity gaps. Since not all particles have the same amount of energy and phase, the particles populate in a region in the phase space. Under adiabatical (slowly varying of external fields) conditions, each particle traces out a closed trajectory in the longitudinal phase space. Following Boltzmann-Ehnenfest theorem the area enclosed by such a trajectory is conserved.

Longitudinal emittance is practically defined by the area of a reference trajectory which encompasses $95 \%$ that of the rest particles' in the bunch. Longitudinal emittance is conserved if adiabaticity holds. Thus, smaller bunch length gives rise to larger energy spread and vice versa. Larger energy spread in the beam, however, demands larger RF cavity gap volts. Therefore, a given RF system prefers a smaller longitudinal emittance for colliding beam experiments. From the point of view to produce short bunches, the smaller the longitudinal emittance the Booster produces the better. A nominal emittance value of $0.3 \mathrm{eVs} / u$ [1] is required for $R H I C$.

\section{Measurements and Results}

A wall current monitor ( $W C M$ ) opens up one door to access to one dimension of the two dimensional longitudinal phase space. The $W C M$ monitors passages of the bunches and maps out the current signals, from which bunch lengths can be measured accordingly. The other dimension, however, can't be measured in a non-parasitic way, we need to utilize the synchrotron equation which depends on the state of the accelerator [2], specifically the $R F$ voltage, $\mathrm{RF}$ frequency or the bending magnetic field, and the rate of change of the bending magnetic field, from which the synchrotron equation is known.

The validity of the synchrotron equation near transition is not a concern for us, since the Booster is operated far away from transition region. We also avoid the ambiguity during bunch coalescing-merging more than two bunches into one bunch-by only make measurements before and after each coalescing.

Since Band II cavities were not yet available, the two Band III cavities $(2.4 \mathrm{MHz}-$ $4.5 \mathrm{MHz}$ ) were used to coalesce the ion beams. The $\mathrm{Au}^{33+}\left(\mathrm{Si}^{8+}\right)$ beams are initially captured and bunched in 48 bunches (24). Each coalescing reduces the bunch numbers by a half, eventually after 4 (3) times of coalescing only 3 bunches are left and extracted to AGS. Each final bunch consists of $\frac{48}{3}\left(\frac{24}{3}\right)$ initial bunches. During the $\mathrm{Au}^{33+}\left(\mathrm{Si}^{8+}\right)$ cycle, these 4 (3) coalescing points divide the cycle into 5 (4) regions, we make measurements at three points in each region. At each point, the bunch lengths, rate of change of magnetic field, cavity gap votage are measured with LeCroy $9474 \mathrm{E}$ Quad $350 \mathrm{MHz}$ oscilloscope; the $R F$ frequency is measure with Hewlett-Packard 5372A Frequency and Time Interval Analyzer. The longitudinal emittance is calculated from measurements for each bunch and normalized for three bunches at each point.

In Figures 1 and 2, each plot corresponds to measurements done at a different date. For a perfect coalescing procedure, the normalized emittance should be a constant throughout the cycle. As expected that each coalescing blows up the emittance to some extent. Note the last coalescing is purposely made worse off, $S i^{8+}$ beams in particular, to facilitate operations in the $A G S$. 


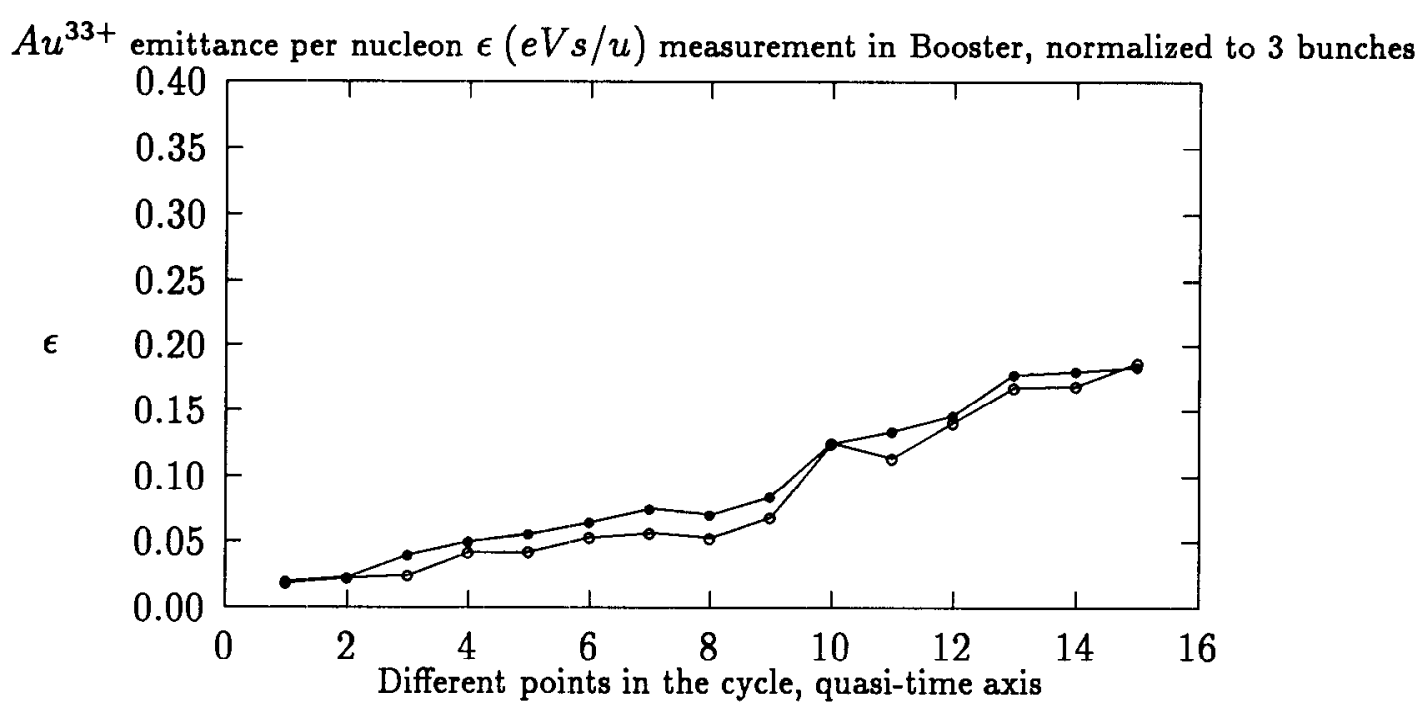

Figure 1: Normalized emittance for three bunches in $A u^{33+}$ cycles.

$S i^{8+}$ emittance per nucleon $\epsilon(e V s / u)$ measurement in Booster, normalized to 3 bunches

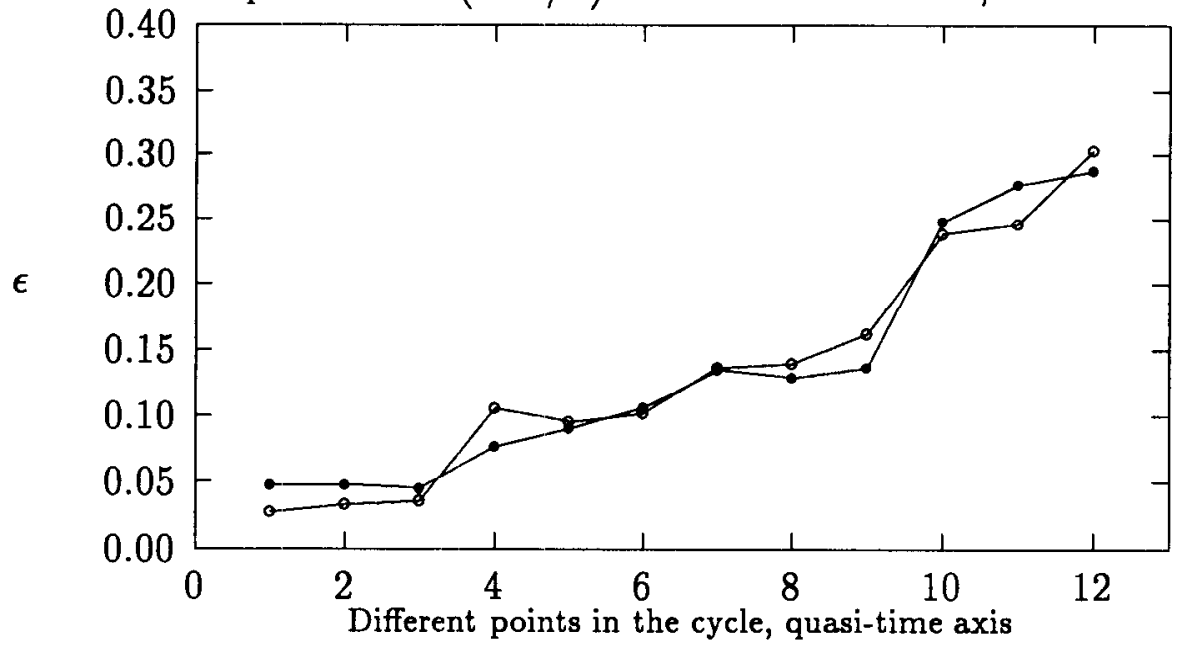

Figure 2: Normalized emittance for three bunches in $S i^{8+}$ cycles. 


\section{A Longitudinal Emittance Monitor?}

The longitudinal emittance is conserved only under ideal adiabatical conditions, anything less stringent than those will make it to grow larger. It is naturally a requirement to inject only those "good" bunches, whose emittances are less than the nominal emittance specified in $R H I C$, into the $R H I C$ rings. It calls for a longitudinal emittance monitor to discriminate against "bad" bunches. Such a proposal is under way.

\section{Acknowledgement}

I would like to express my deep gratitude to J. M. Brennan for his guidance throughout the project. Many thanks also go to J. DeLong, T. Hayes, D. Gassner and W. Zhang for their help in operating the instruments, to J. Rose for his comments on the manuscript.

\section{References}

[1] Conceptual design of the relativistic heavy ion collider. BNL 52195, May 1989.

[2] I. Gumowski. Width, height and area. MPS/Int. RF 67-1, CERN, 1967. 